\title{
Non-rigid registration of breast surfaces using the laplace and diffusion equations
}

\author{
Rowena E Ong, Jao J Ou, Michael I Miga*
}

* Correspondence: michael. miga@vanderbilt.edu Department of Biomedical Engineering, Vanderbilt University, Nashville, TN, USA

\begin{abstract}
A semi-automated, non-rigid breast surface registration method is presented that involves solving the Laplace or diffusion equations over undeformed and deformed breast surfaces. The resulting potential energy fields and isocontours are used to establish surface correspondence. This novel surface-based method, which does not require intensity images, anatomical landmarks, or fiducials, is compared to a gold standard of thin-plate spline (TPS) interpolation. Realistic finite element simulations of breast compression and further testing against a tissue-mimicking phantom demonstrate that this method is capable of registering surfaces experiencing $6-36 \mathrm{~mm}$ compression to within a mean error of $0.5-5.7 \mathrm{~mm}$.
\end{abstract}

\section{Background}

As breast cancer is estimated to kill over 40,600 people and be diagnosed in more than 194,000 in 2009 [1], its detection and treatment is an important area of scientific research. Many novel techniques to aid in tumor detection are being developed that exploit the difference in physical properties between healthy and cancerous tissue. Some of these techniques measure the optical, electrical, or elastic properties of tissue, such as near-infrared tomography [2], electrical impedance tomography [3], ultrasound elastography [4], magnetic resonance elastography [5,6], and modality-independent elastography (MIE) [7-9]. Regardless of the means of data acquisition, it is important to recognize and account for the soft-tissue deformation mechanics of the breast during any analysis.

Previous work in non-rigid registration methods can be broadly categorized as being feature-based or intensity-based. Feature-based methods use only the geometric information extracted from an image, such as a polygonal mesh constructed from a segmented image. Examples of feature-based methods include symmetric closest point [10], robust point matching [11], methods involving implicit functions [12], and finite element modeling [13]. One type of feature-based registration involves the use of splines to interpolate the displacements between tracked control points. Polynomial splines, B-splines, and thin-plate splines (TPS) are among the most commonly used. However, the difficulty with using any type of spline is determining accurate displacements at the control points; the displacements must either be tracked with fiducial markers or estimated using another method such as in [14].

In contrast, intensity-based methods utilize the intensities in the image volume, sometimes in addition to the geometric information, to register two images. Rueckert $[15,16]$ proposed a method to maximize image similarity and preserve smoothness, while a similar

(c) 2010 Ong et al; licensee BioMed Central Ltd. This is an Open Access article distributed under the terms of the Creative Commons Attribution License (http://creativecommons.org/licenses/by/2.0), which permits unrestricted use, distribution, and reproduction in any medium, provided the original work is properly cited. 
volume-preserving optimization method was developed by Rohfing [17]. Tanner [18] employed a free-form b-spline deformation to maximize image similarity in a volumepreserving cost function, similar to Rueckert. The registration algorithm was validated by taking clinical dynamic contrast enhanced MR images, deforming them using a biomechanical FEM model and then calculating a TRE. Optical flow [19] and fluid flow [20] techniques have also been used for breast image registration. Unfortunately, like all optimization schemes, intensity-based methods are subject to the need for good initialization and vulnerable to local minima. In addition, intensity information may not be readily available for some applications such as near-infrared breast tomography, electrical impedance tomography, microwave tomography, or mechanical imaging. Even if intensity images are available, they may be subject to geometric distortions or contrast changes.

For the particular application of registering breast surfaces having undergone a quasi-static mechnical compression, our prior work has indicated that fiducial-based spline interpolations, while powerful, do not translate well with experimentation on tissue and substances not amenable to the fixation of physical markers. Likewise, intensity-based methods may not be desirable due to computational expense or the unavailability of suitable intensity images. Therefore, in an attempt to balance the best attributes of these classes of methods, we present a semi-automated method that does not rely on either control points or explicit knowledge of the internal image intensity pattern. This is accomplished by using the Laplace or diffusion equations to calculate equivalent surface energy distributions in order to estimate a generalized displacement field. The accuracy of the method was evaluated by comparison to our internal gold standard of a thin-plate spline interpolation method [21] in both biomechanical simulations and experimental deformations on breast phantoms.

\section{Methods}

\section{PDE-based registration}

The basic premise of this work was to evaluate whether the equivalent potential energy distributions modeled by a partial differential equation (PDE) over an undeformed ("source") surface and a deformed ("target") surface could be used to determine correspondence between the two surfaces. For our specific method, finite element models of two classic PDEs (Laplace's equation and the diffusion equation) were solved independently over the source and target surfaces using the Galerkin method of weighted residuals with Lagrange polynomial interpolation [22]. Equivalent potential energy isocontours were calculated and matched on a closest-point basis, and from this correspondence the final displacements at all mesh nodes were interpolated.

Laplace's equation is most commonly used to describe potential flow problems in thermal, fluid, and electrostatic systems and is given by

$$
\nabla \bullet(-\sigma \nabla \Phi)=0
$$

where $\Phi$ represents the potential and $\sigma$ describes the spatially varying conductivity. The diffusion equation, which utilizes a time-varying component, is given by

$$
\frac{\partial \Phi}{\partial \mathrm{t}}=\nabla \bullet(\alpha \nabla \Phi)
$$

where $\Phi$ represents the potential and $\alpha$ is the diffusion coefficient. 
To solve Laplace's equation (1), Dirichlet (Type I) boundary conditions were set to allow "flow" from a high- to low-potential area (Figure 1, Step 2). Specifically, nodes in the nipple and chest wall area were given boundary potential values of 1 and 0 , respectively, and the conductivity $\sigma$ was set to unity. The diffusion equation (2) was solved by temporally stepping the FEM solution using a fully explicit forward Euler scheme. For each data set utilized in this study, optimal ranges were empirically determined for time step ([1e-7, 8e-7]) and final time ([0.005, 0.01]). A pure Neumann (Type II, no flux) boundary condition was prescribed at the chest wall, and the potential field was allowed to propagate from a source located at the nipple. The diffusion coefficient $\alpha$ was set to unity. The diffusing front was stopped once the potential field reached the chest wall.

The solutions $\Phi_{\text {source }}$ and $\Phi_{\text {target }}$ obtained from the PDEs were used to establish correspondence between the source and target nodes. This involved two distinct processes: finding point correspondence between isocontours of $\Phi_{\text {source }}$ and $\Phi_{\text {target }}$ and interpolating the displacements at these isocontour points to all nodes in the mesh. In the first step, isocontours were extracted from $\Phi_{\text {source }}$ and $\Phi_{\text {target }}$ for a set of selected isovalues (Figure 2, Step 4). The correspondence between the source and target isocontour points was determined by aligning the contours by their centroids and using the symmetric closest point (SCP) algorithm (Figure 2, Step 5. See Figure 3 for detailed description of SCP). In the second step, the displacement vectors at the source isocontours points were interpolated to all source nodes.

The method can be summarized in the following steps (Figures 1, 2):

1. Obtain the undeformed source mesh and deformed target mesh that define a breast surface before and after deformation.

2. Assign boundary conditions at nipple and/or chest wall nodes

3. Solve the PDE (diffusion or Laplace) over the source and target meshes using FEM.

4. Extract isocontours on the source and target surfaces.

5. Determine point correspondence between source and target isocontours using SCP (Figure 3).

6. Interpolate displacements at source isocontours to all source nodes.

\section{TPS registration}

As noted earlier, there are numerous methods of spline-based interpolation. TPS interpolation was chosen in part because it is a standard, well-characterized method in the literature [20] that has been successfully used in many non-rigid registration applications. Because it does not require a regular grid, the effects of changing a control point are relatively localized. The overall registration is achieved by the warping of a hypothetical thin sheet of metal using a series of radial basis functions based on a number of fixed control points. The global deformation field was then interpolated back to the surface node coordinates of the finite element mesh. The displacement vector at point $(x, y, z)$ is therefore described by the following linear system: 
1. Obtain the undeformed source (left) and deformed target mesh (right) that define a breast surface before and after deformation.

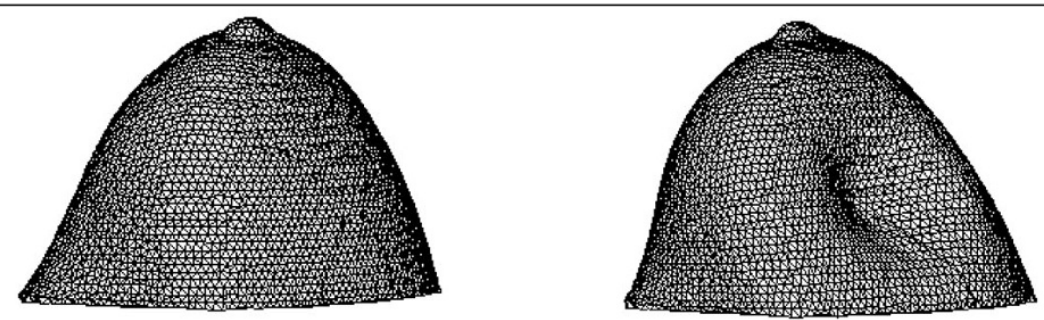

2. Assign boundary conditions at the nipple and chest wall nodes on the source (left) and target (right) meshes. To solve the Laplace equation, assign type 1 boundary conditions at nipple nodes $(\Phi=1)$ and at chest wall nodes $(\Phi=0)$. To solve the diffusion equation, assign type 1 boundary conditions at nipple $(\Phi=1)$ and no-flux conditions at chest wall nodes ( $d \Phi / \mathrm{d} \mathbf{X}=0$ ).
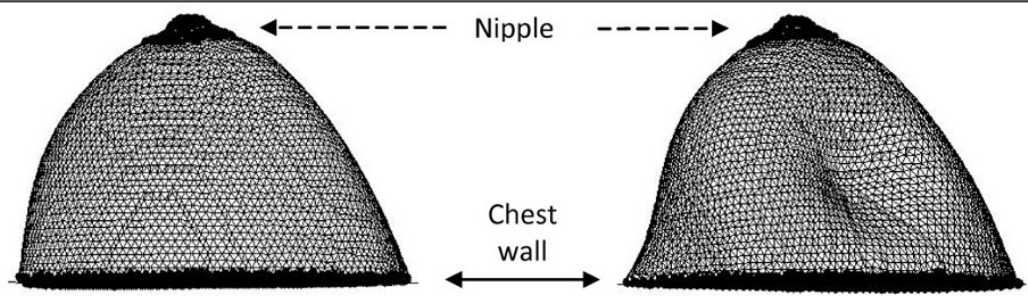

3. Solve the Laplace or diffusion equations over the source (left) and target (right) meshes using FEM. The solutions to the Laplace or diffusion equation $\Phi_{\text {source }}$ and $\Phi_{\text {target }}$ describe the potential at each surface node (indicated by the shading in the figures below).
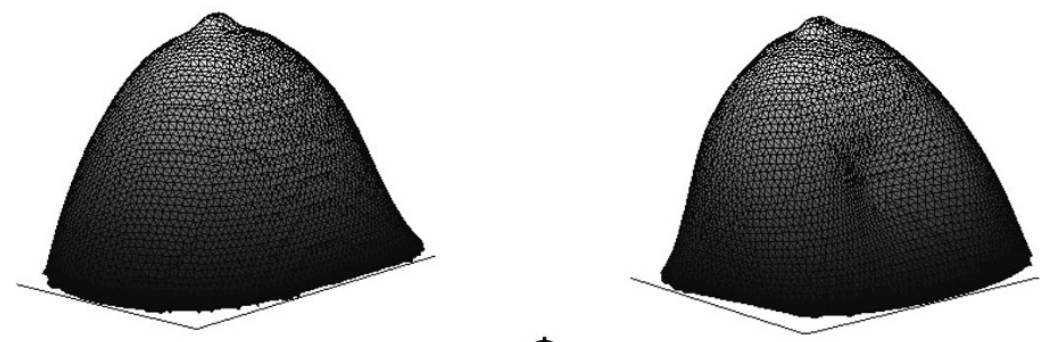

$\Phi$

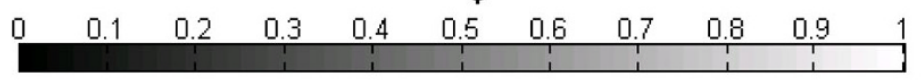

Figure 1 Summary of the PDE-based registration methods (steps 1-3).

$$
f(x, y, z)=a \cdot x+b \cdot y+c \bullet z+\sum_{i=1}^{N} F_{i} \cdot r_{i}^{2} \bullet \ln r_{i}^{2}
$$

where

$$
\mathbf{r}_{\mathbf{i}}^{2}=\left(\mathbf{x}-\mathbf{X}_{\mathbf{i}}\right)^{2}+\left(\mathbf{y}-\mathbf{Y}_{\mathbf{i}}\right)^{2}+\left(\mathbf{z}-\mathbf{Z}_{\mathbf{i}}\right)^{2}
$$

with constraints

$$
\sum_{i=0}^{N} F_{i}=0
$$


4. Extract isocontours on the source (left) and target (right) surfaces.
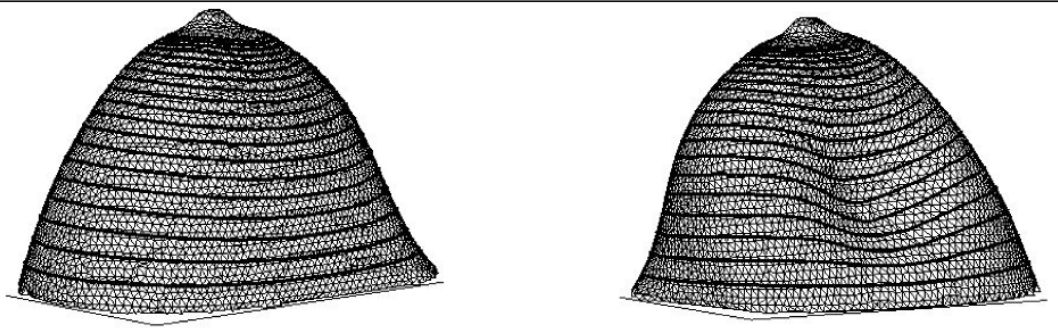

5. Determine point correspondence between source and target isocontours using SCP method. For each isovalue, the SCP method will return a mapping between the source isocontour (left) and target isocontour (right) points. The vectors describing the displacement (bottom, black dotted lines) between the source and target isocontour points can then be calculated by a simple subtraction.

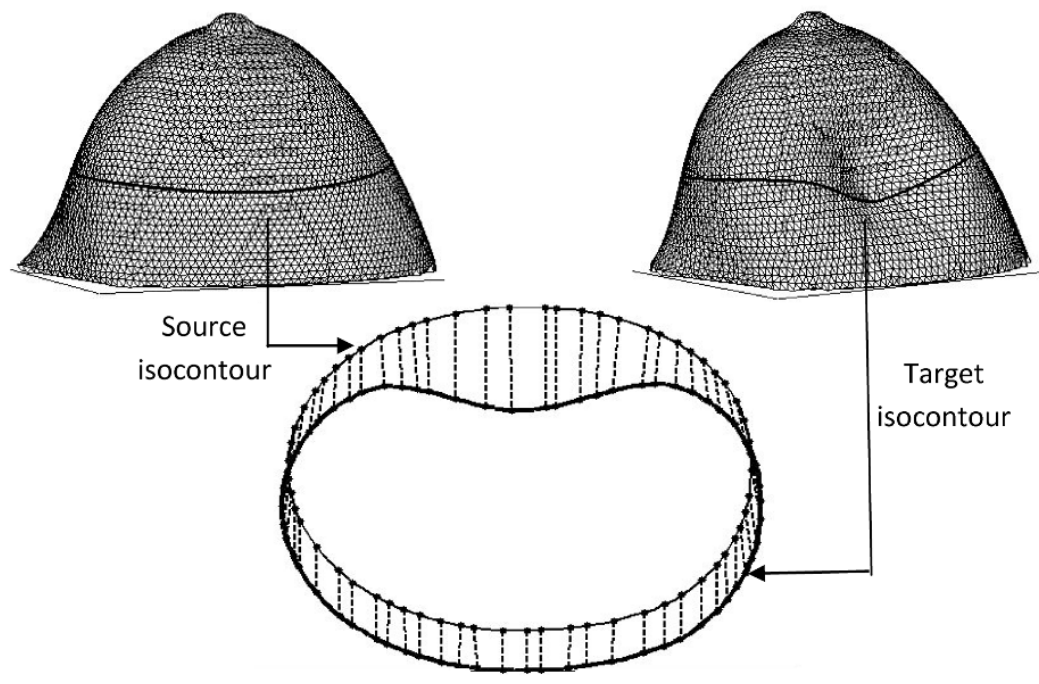

6. Interpolate displacements from source isocontour points to all source surface nodes.

Figure 2 Summary of the PDE-based registration methods (steps 4-6).

$$
\begin{aligned}
& \sum_{i=0}^{N} X_{i} F_{i}=0 \\
& \sum_{i=0}^{N} Y_{i} F_{i}=0 \\
& \sum_{i=0}^{N} Z_{i} F_{i}=0
\end{aligned}
$$

where $\mathbf{X}_{\mathbf{i}}, \mathbf{Y}_{\mathbf{i}}, \mathbf{Z}_{\mathbf{i}}$ are the coordinates of the control points, $\mathbf{N}$ is the number of control points, and $\mathbf{a}, \mathbf{b}, \mathbf{c}$, and $\mathbf{F}$ are scalar weighting factors. 


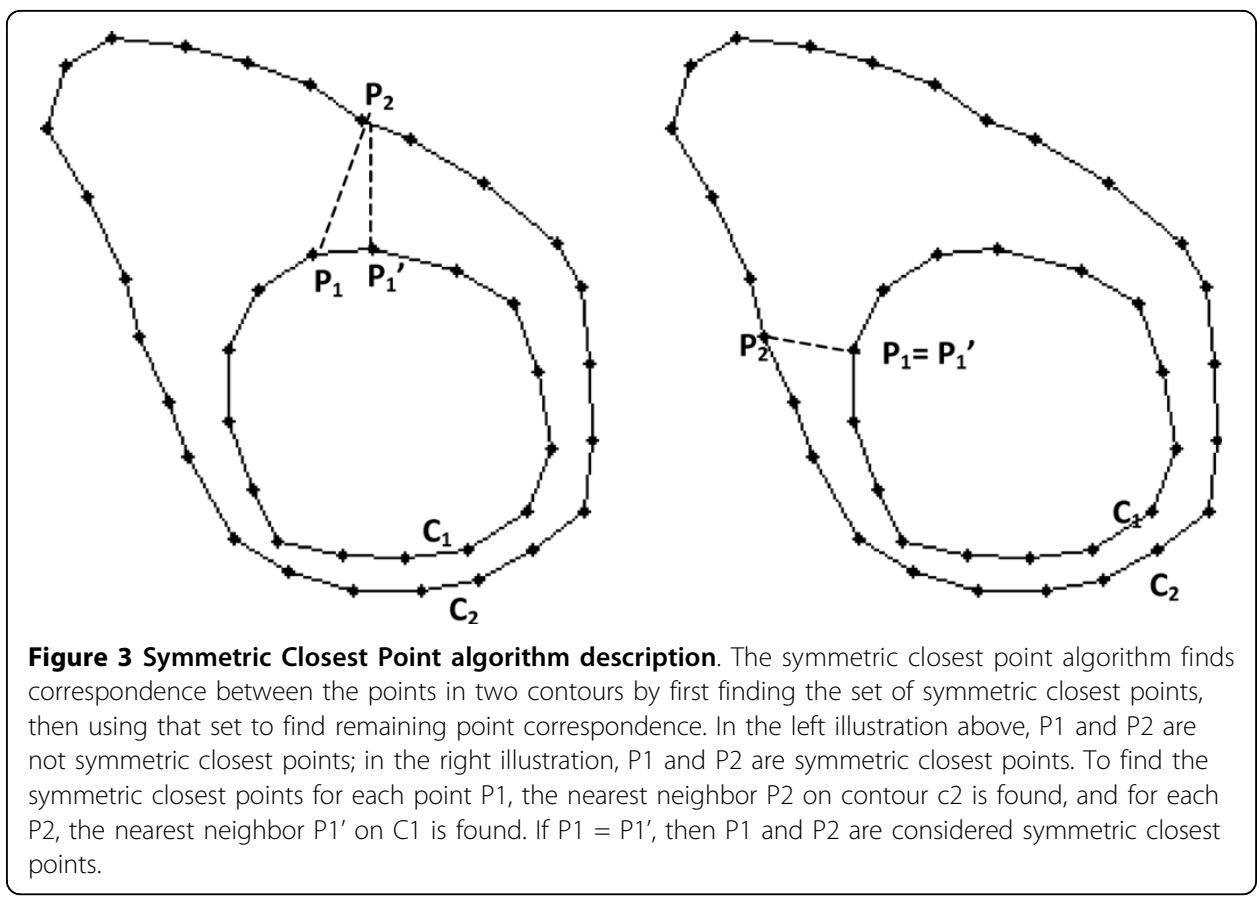

\section{Simulation experiments}

To assess the accuracy of the PDE-based and TPS methods described above, breast surfaces deformed using a biomechanical model were registered and the simulated displacements were used to calculate the registration error. A CT image volume of a pendant human breast $\left(256 \times 256 \times 130\right.$, voxel size $\left.0.6 \mathrm{~mm}^{3}\right)$ and an MR image volume of a pendant breast from a different patient $\left(256 \times 256 \times 98\right.$, voxel size $1.0 \mathrm{~mm}^{3}$, 3D T1weighted fat-nulling inversion pulse sequence) were obtained. Both image volumes were segmented using ANALYZE 6.0 (Mayo Clinic, Rochester, MN), and triangular source meshes consisting of 6,313 and 3,942 nodes, respectively, were constructed.

Target surfaces were created by deforming the source surfaces using a finite element model of the breast as a linear elastic, Hookean solid under localized compression. The nodes along the chest wall were made to be fixed (Type I) and a Gaussian-shaped stress distribution (Type II) applied to the lateral surface of the breast. To provide a challenge to our registration method, the shape and magnitude of the applied stress was varied. First, the CT source surface was deformed using a circular contact area with a maximum displacement of approximately $33 \mathrm{~mm}$. In the second simulation, a rectangular contact area was used with a maximum displacement of $13 \mathrm{~mm}$. Finally, in the third simulation, the MR source surface was deformed assuming a rectangular contact area and a maximum displacement of $6 \mathrm{~mm}$.

In addition to running the Laplace and diffusion registrations, TPS registration was performed for further comparison. Because the accuracy of the TPS method varies based on the number and distribution of control points, two different sets of registrations were performed. In the first analysis, a uniform distribution of control points over the breast surface was selected using a k-means algorithm. The number of control points was varied, and for a particular number of control points desired, 20 different configurations were selected to account for the initial random seeding of the $k$-means 
clustering algorithm. In the second analysis, a high number of control points in the deformed region (the part of the surface in contact with the simulated inflation bladder) and a lower number over the rest of the surface was used. Similarly, the error was calculated for a varying number of control points and configurations.

To assess the accuracy of the registration methods, the target registration error (TRE) was calculated as the Euclidean distance between the coordinates determined by non-rigid registration and the true target points. Because of the controlled modelbased deformation, every node on the surface had a known correspondence from source to target surfaces, allowing for individual TRE determinations along with evaluation of mean and max error for the surface as a whole.

\section{Phantom experiments}

To test the registration methods on real-world data, a semi-anthropomorphic breast phantom was fabricated from an $8 \% \mathrm{w} / \mathrm{v}$ solution of polyvinyl alcohol (Flinn Scientific, Batavia, IL) that was frozen at $-37^{\circ} \mathrm{C}$ in a plastic mold for 16 hours and thawed to ambient room temperature. Thirty-four $1-\mathrm{mm}$ stainless steel ball bearings were implanted directly under the surface of the resulting tissue-mimicking cryogel to act as fiducials. The phantom was then placed inside a custom-built acrylic chamber designed to deliver compression by means of an air bladder positioned against the surface of the phantom (Figure 4).

CT images $(512 \times 512 \times 174,0.54 \times 0.54 \times 1 \mathrm{~mm}$ voxel size $)$ were acquired with the phantom subjected to three different states of mechanical deformation (undeformed, $50 \%$ of maximum bladder pressure, and full inflation of approximately $200 \mathrm{~mm} \mathrm{Hg}$ ).

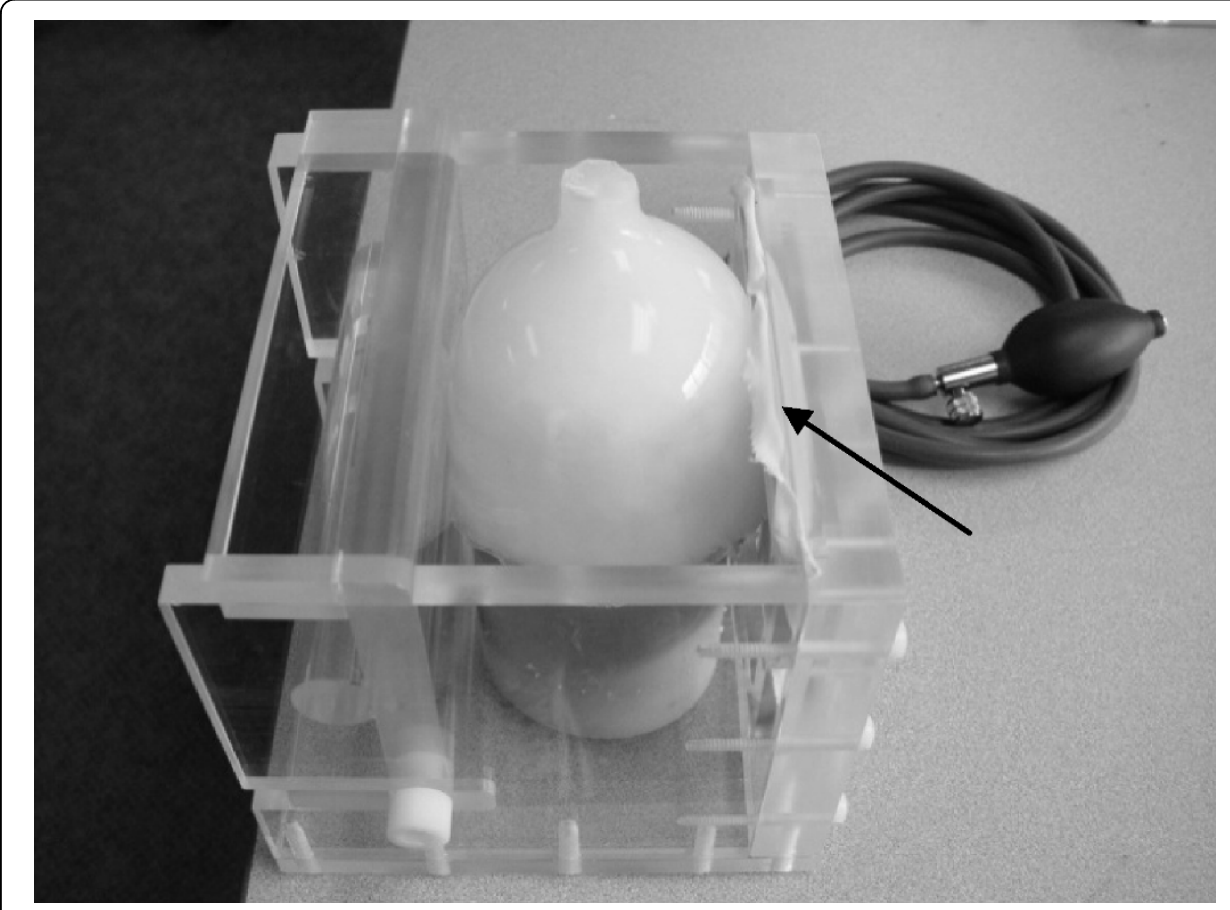

Figure 4 Breast compression chamber. Experimental system for applying compression to breast phantom. A polyvinyl alcohol cryogel is placed within a Plexiglas chamber with its surfaces held in place against the walls. Compression is delivered through an air bladder (arrow) inflated manually through a bulb adapted from a standard sphygmomanometer. 
Triangular surface meshes were obtained by segmentation of the image volumes using the surface extraction tools in ANALYZE, and the coordinates of the fiducial centroids localized. These meshes contained 8,127, 6,777, and 8,260 nodes, respectively. The Laplace, diffusion, and TPS methods were then used to register the phantom surface meshes as described above. Accuracy was assessed by calculating the TRE at the embedded fiducials. For the TPS method, 33 of the fiducials were used as control points in the interpolation and the remaining fiducial was reserved for calculating the TRE. To assess the error over the entire surface, the TPS registration was repeated in a "leave one out" scheme, each time using a different fiducial to calculate the error, with the final TRE representing the average over all trials.

\section{Results}

\section{Simulation experiments}

As described in Section 2.3, the Laplace and diffusion methods were used to register the breast surfaces deformed by the three simulated compressions. For each simulation, the accuracy of the Laplace and diffusion methods was assessed by calculating the TRE at each node and comparing it with the TRE obtained using the TPS method (Figure 5). The results (Table 1) indicate that the Laplace and diffusion methods could register breast surfaces with up to $33 \mathrm{~mm}$ of compression with errors of $0.5-1.8 \mathrm{~mm}$, while the TPS method generated errors of up to $0.44 \mathrm{~mm}$.

To evaluate how the number and placement of fiducials affects TRE, the TPS registration was performed for differing numbers of fiducials, placed in uniform (Figure 6) and non-uniform (Figure 7) fiducial distributions. The results of the TPS registrations indicate that when a uniform fiducial distribution is used, the error decreases as the number of fiducials is increased. However, increasing the fiducial number over about 40 does not seem to result in a significant error reduction. For a non-uniform distribution, the error does not seem to decrease as the number of fiducials outside the contact region is increased. In other words, the same amount of error could be obtained using a smaller number of fiducials, as long as more control points are placed in the contact region of the simulated compression bladder.

\section{Phantom experiment}

The Laplace and diffusion methods were used to determine point correspondence between the uncompressed and compressed surfaces of a breast phantom. The results were validated by calculating the TRE at 34 fiducials located directly below the surface of the phantom. For comparison, TPS registration was used to interpolate the displacements at the fiducials to all surface nodes and the TRE was calculated as described in Section 2.4. The results for a 50 and $100 \%$ compression (with a maximum displacements of about $20 \mathrm{~mm}$ and $36 \mathrm{~mm}$, respectively) are shown in Table 2.

\section{Discussion}

While neither the Laplace nor diffusion PDE-based methods were able to surpass the performance of a well-executed TPS-based interpolation, the results are encouraging overall as a first attempt of a method that does not require fiducials.

One area of further development with regards to the Laplace equation method is in determining specific regions to which boundary conditions are assigned. For the geometry of the breast, the nipple and chest wall areas were relatively evident and easily 


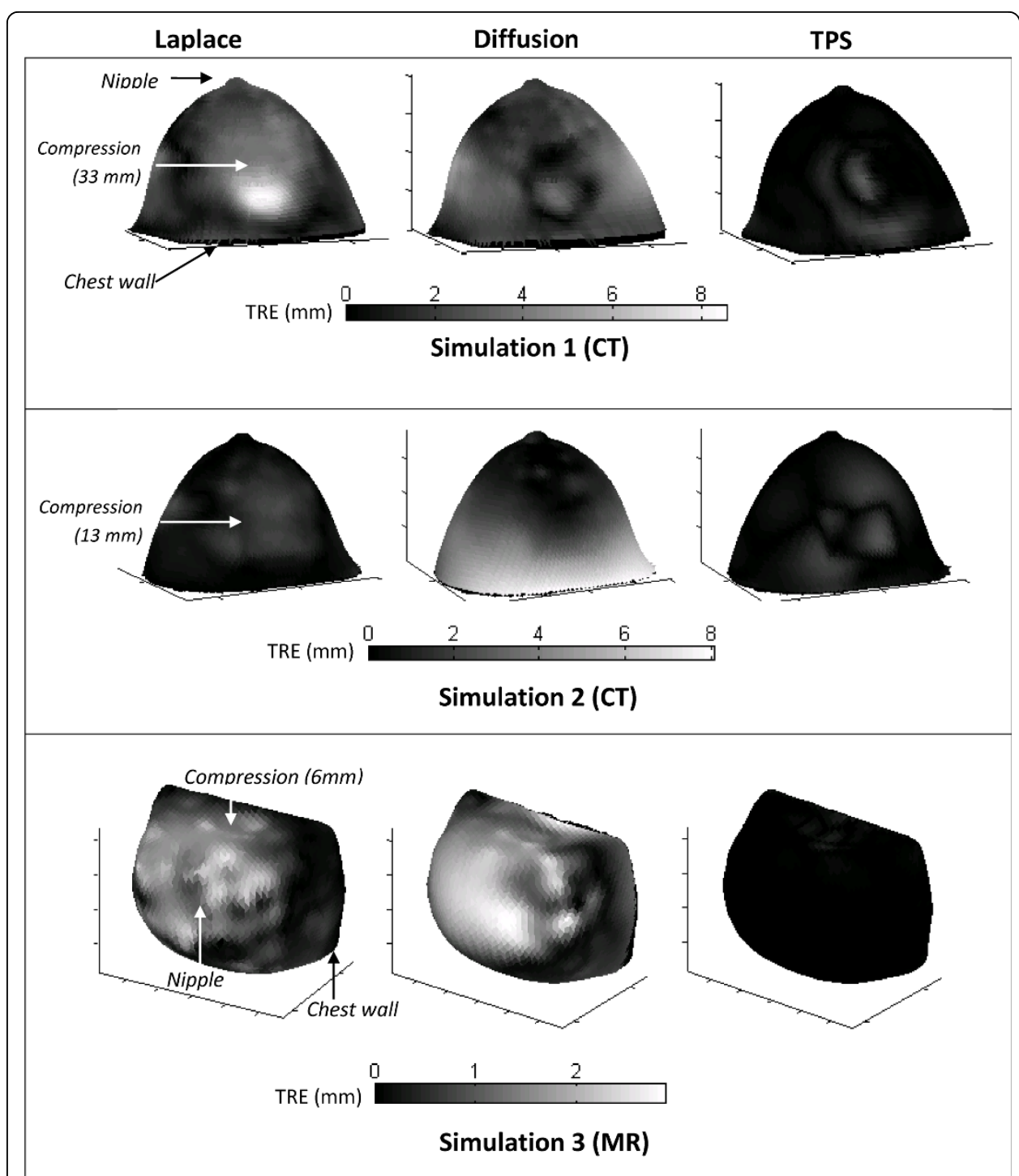

Figure 5 Simulation registration error. Error when breast surfaces deformed by three simulations were registered using the Laplace (left column), diffusion (middle column), and TPS (right column) registrations. The TPS registration method had lower error than the Laplace or diffusion methods in all three simulations.

Table 1 Simulation registration error

\begin{tabular}{|c|c|c|c|c|c|c|}
\hline & \multicolumn{2}{|c|}{$\begin{array}{l}\text { Simulation } 1(\mathrm{CT})(33 \mathrm{~mm} \\
\text { displacement) }\end{array}$} & \multicolumn{2}{|c|}{$\begin{array}{l}\text { Simulation } 2 \text { (CT) } \\
\text { (13 mm displacement) }\end{array}$} & \multicolumn{2}{|c|}{$\begin{array}{l}\text { Simulation } 3 \text { (MR) } \\
\text { (6 mm displacement) }\end{array}$} \\
\hline & $\begin{array}{l}\text { Max TRE } \\
(\mathrm{mm})\end{array}$ & $\begin{array}{l}\text { Mean TRE } \\
(\mathrm{mm})\end{array}$ & $\begin{array}{l}\text { Max TRE } \\
(\mathrm{mm})\end{array}$ & $\begin{array}{l}\text { Mean TRE } \\
(\mathrm{mm})\end{array}$ & $\begin{array}{l}\text { Max TRE } \\
(\mathrm{mm})\end{array}$ & $\begin{array}{l}\text { Mean TRE } \\
(\mathrm{mm})\end{array}$ \\
\hline Laplace & 8.5 & 1.6 & 2.6 & 0.53 & 2.5 & 0.48 \\
\hline Diffusion & 6.7 & 1.8 & 8.0 & 1.5 & 2.9 & 0.61 \\
\hline$\overline{\text { TPS }^{*}}$ & 3.1 & 0.44 & 2.6 & 0.26 & 0.6 & 0.033 \\
\hline
\end{tabular}

Max and mean TRE when the Laplace, diffusion, and TPS methods were used to register breast surfaces deformed by three simulations.

*TPS registration using 40 uniformly distributed fiducials for the CT simulations and 60 for the MR simulation. 


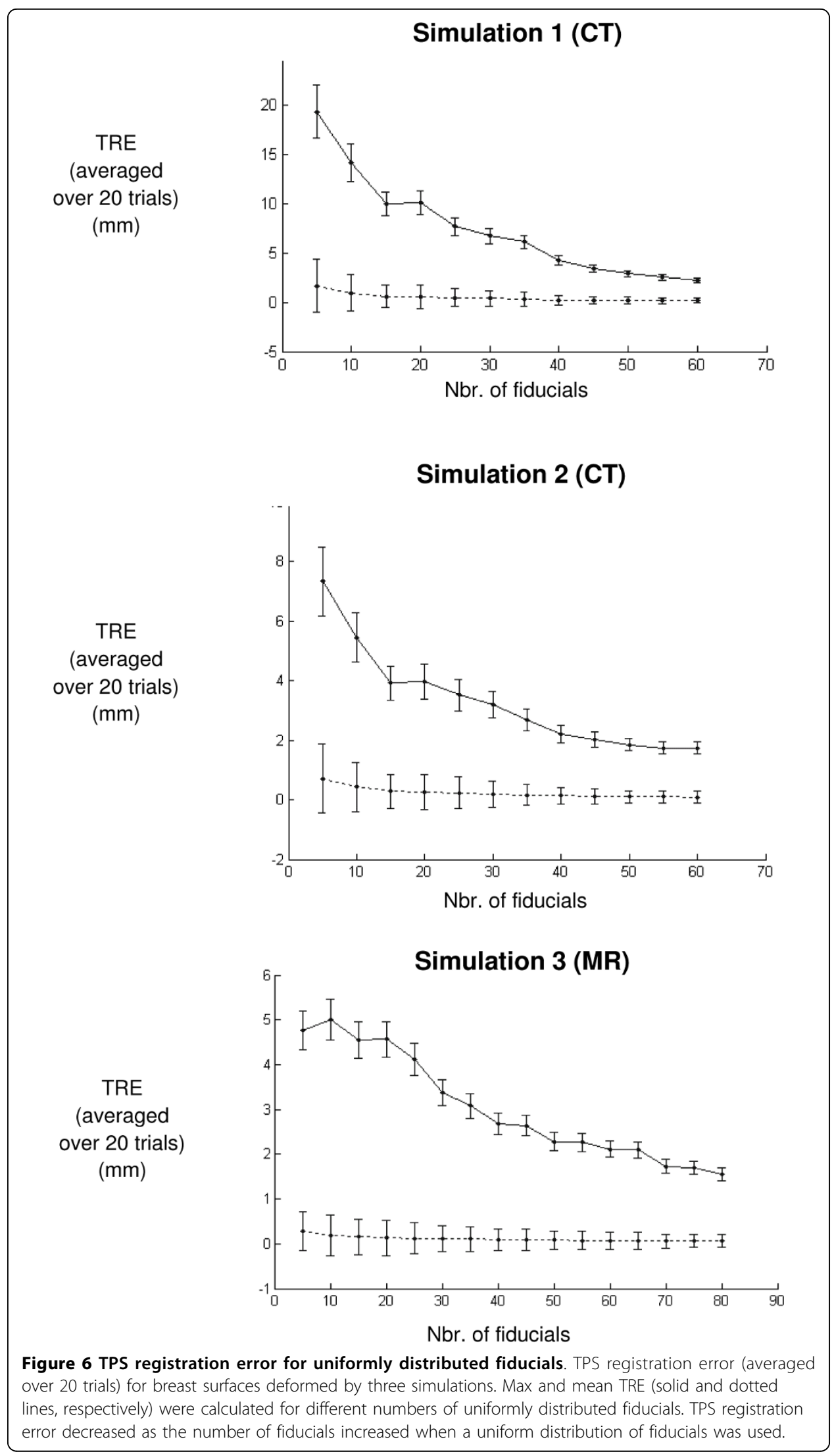




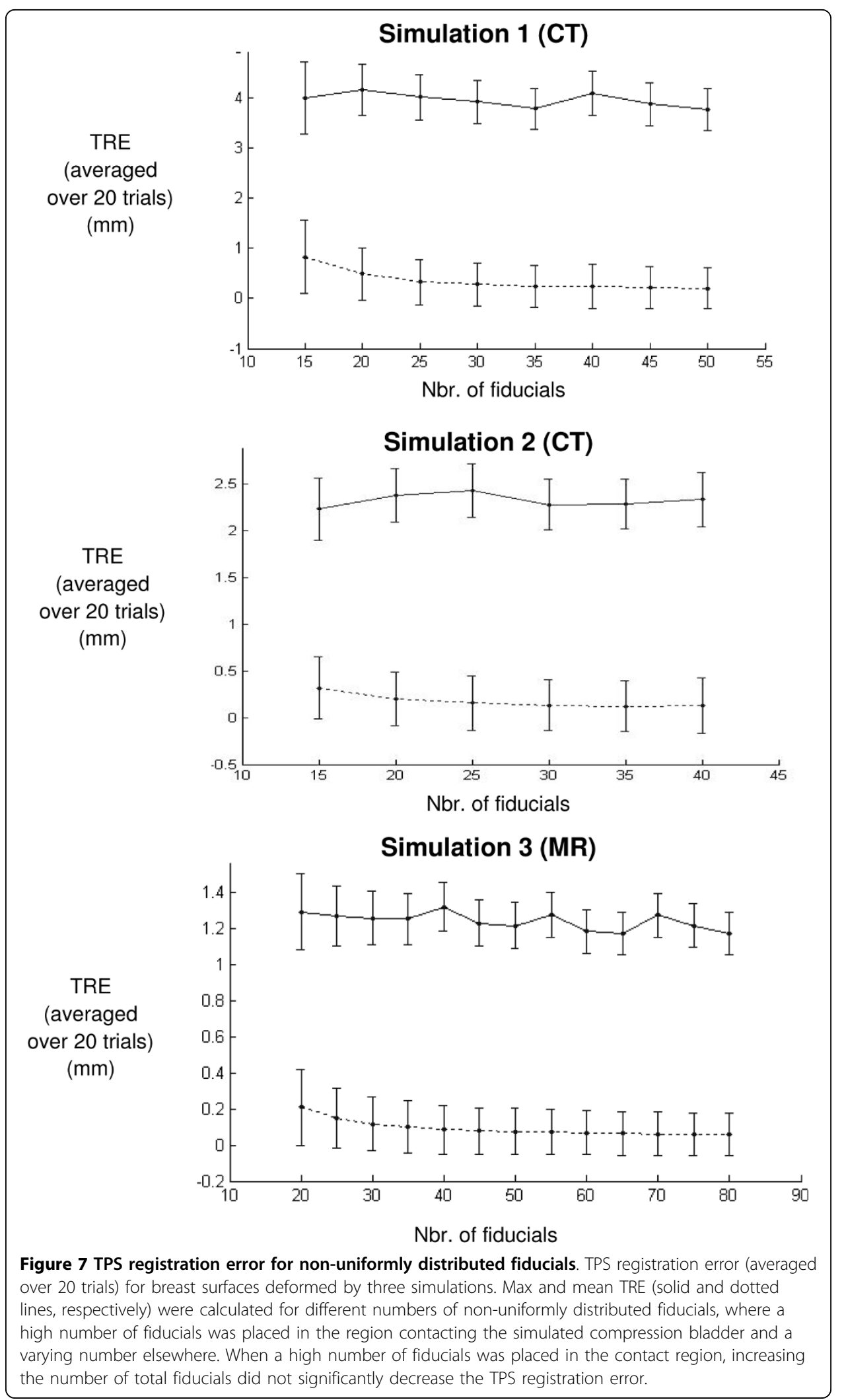


Table 2 Phantom registration error

\begin{tabular}{lllll}
\hline & \multicolumn{2}{l}{$\begin{array}{l}\text { Phantom: } \mathbf{5 0 \%} \text { compression } \\
(\mathbf{2 0} \mathbf{~} \mathbf{m} \text { displacement) }\end{array}$} & \multicolumn{2}{l}{$\begin{array}{l}\text { Phantom: } \mathbf{1 0 0 \%} \text { compression } \\
\mathbf{( 3 6} \mathbf{~} \mathbf{m} \text { displacement) }\end{array}$} \\
\cline { 2 - 5 } & Max TRE $(\mathbf{m m})$ & Mean TRE $(\mathbf{m m})$ & Max TRE $(\mathbf{m m})$ & Mean TRE $(\mathbf{m m})$ \\
\hline Laplace & 8.6 & 3.4 & 15.3 & 6.3 \\
\hline Diffusion & 6.8 & 2.7 & 13.6 & 5.7 \\
\hline TPS * & 3.4 & 1.1 & 5.1 & 1.7 \\
\hline
\end{tabular}

Error for different registration methods tested on the breast phantom at 50\% and $100 \%$ compression.

* TPS registration was performed using 33 fiducials and 1 fiducial to calculate TRE. The TRE was averaged over 34 trials, where each trial used a different fiducial to calculated TRE.

set; however, proper selection of these regions is important because the implicit correspondence between these regions determines the potential energy distribution and therefore correspondence for the rest of the surface.

While we do not perform a direct sensitivity analysis for boundary condition selection, previous work indicates that as long as the segmentation and resulting boundary condition error is below a certain threshold, reasonable results may be obtained. In [9], the PDE registration methods were used in a breast elastography application, and a boundary condition sensitivity analysis was performed. Reasonable results were obtained as long as the average error over all boundary condition nodes was below 0.5 voxels. Although a direct sensitivity analysis may be desirable, we feel this threshold may indicate the acceptable error for our PDE registration methods.

Some factors to consider in the diffusion method are the parameters controlling the behavior of the diffusion front over the breast surface. Careful selection of these parameters therefore enabled the diffusion-based method to outperform the Laplace method in certain cases. We believe this advantage may be related to the fact that the diffusion method requires fewer initial selections of boundary conditions to generate potential flow as compared to the Laplace method.

Although the gold standard TPS method outperformed the Laplace and diffusion methods, there are several factors to consider. The gold standard TPS interpolation method requires multiple points of constraint and is highly dependent on their number and placement. When a uniform distribution is used, the error decreases as the number of fiducials is increased but with diminishing returns. It should also be noted that to attain comparable TRE values, the non-uniform fiducial distribution requires fewer control points. The analysis of this behavior is illuminating in providing a set of benchmarks for future development of the PDE-based methods.

The literature is replete with registration methods developed for 2D and 3D mammographic applications, with a relative preponderance towards intensity-based methods. However, conventional intensity images may not be available for some applications, and even if available, they may difficult to utilize due to geometric distortion or contrast changes. The novel surface-based method we have presented may provide a suitable alternative for situations where intensity analysis is not amenable due to unavailability, computational complexity, unsuitable contrast, or imaging artifacts.

In further consideration of the accuracy of our method, we reviewed the excellent recent work presented in [18]. In this work, twelve different types of deformations were modeled ranging from $5-10 \mathrm{~mm}$. For the optimal registration parameters, the average TRE was reported to be $0.45 \mathrm{~mm}$ with a maximum TRE across a series of data 
sets of approximately 4-6 mm. While our results are not directly comparable, we note that the simulations with the closest representative applied deformations of 6 and 13 $\mathrm{mm}$ in magnitude had a mean TRE of 0.48 to 0.53 and $\max \mathrm{TRE}<3 \mathrm{~mm}$. We feel that this is reassuring that our method can perform at this level of accuracy without the prescribed use of either fiducials or image intensity.

\section{Conclusions}

A novel surface-based non-rigid registration method has been developed for this work and compared to a relative gold standard of thin-plate spline interpolation. The results indicate that the Laplace and diffusion methods can accurately register breast surfaces that have experienced a wide range of physical deformation to within mean errors ranging from 0.5 - $5.7 \mathrm{~mm}$. Although these PDE-based methods did not perform as accurately as the control, they may be viable registration techniques when fiducials are not available and image intensity comparison is not required.

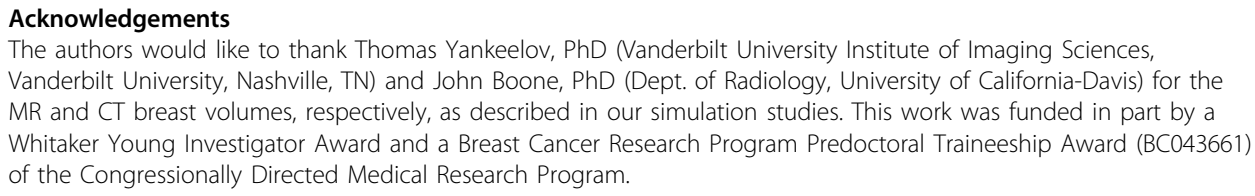

\section{Authors' contributions}

REO performed the simulation and phantom experiments, analyzed the data, and drafted the manuscript. JJO contributed significantly to the experimental design and acquisition of data, as well as providing valuable revisions to the manuscript. MIM was responsible for the initial conception of this project, much of the experimental design, writing the FEM modeling programs, and providing revisions to the manuscript. All authors have approved this final draft of the manuscript.

\section{Competing interests}

The authors declare that they have no competing interests.

Received: 25 June 2009 Accepted: 12 February 2010 Published: 12 February 2010

References

1. Cancer facts and figures. American Cancer Society 2009 [http://www.cancer.org/downloads/STT/500809web.pdf].

2. Brooksby BA, Dehghani H, Pogue BW, Paulsen KD: Near-infrared (NIR) tomography breast image reconstruction with a priori structural information from MRI: algorithm development for reconstructing heterogeneities. IEEE Journal of Selected Topics in Quantum Electronics 2003, 9:199-209.

3. Cherepenin V, Karpov A, Korjenevsky A, Kornienko V, Mazaletskaya A, Mazourov D, Meister D: A 3D electrical impedance tomography (EIT) system for breast cancer detection. Physiological Measurement 2001, 22:9-18.

4. Ophir J, Cespedes I, Ponnekanti H, Yazdi Y, Li X: Elastography - a quantitative method for imaging the elasticity of biological tissues. Ultrasonic Imaging 1991, 13:111-134.

5. McKnight AL, Kugel JL, Rossman PJ, Manduca A, Hartmann LC, Ehman RL: MR elastography of breast cancer: preliminary results. AJR Am J Roentgenol 2002, 178(6):1411-1417.

6. Sinkus $R$, Tanter M, Xydeas T, Catheline S, Bercoff J, Fink M: Viscoelastic shear properties of in vivo breast lesions measured by MR elastography. Magn Reson Imaging 2005, 23(2):159-165.

7. Miga Ml: A new approach to elastography using mutual information and finite elements. Physics in Medicine and Biology 2003, 48:467-80

8. Washington CW, Miga M: Modality independent elastography (MIE): a new approach to elasticity imaging. IEEE Transactions on Medical Imaging 2004, 23:1117-28.

9. Ou JJ, Ong RE, Yankeelov TE, Miga Ml: Evaluation of 3D modality-independent elastography for breast imaging: a simulation study. Physics in Medicine and Biology 2008, 53(1):147-163.

10. Papademetris $X$, Sinusas AJ, et al: Estimation of 3-D left ventricular deformation from medical images using biomechanical models. IEEE Transactions on Medical Imaging 2002, 21(7):786-800.

11. Chui $\mathrm{HL}$, Rangarajan $\mathrm{A}$ : A new point matching algorithm for non-rigid registration. Computer Vision and Image Understanding 2003, 89(2-3):114-141.

12. Dinh HQ: Implicit Shapes: Reconstruction and Explicit Transformation. PhD Dissertation, Georgia Institute of Technology, College of Computing 2002.

13. Roose $\mathrm{L}$, Mollemans $\mathrm{W}$, et al: Biomechanically based elastic breast registration using mass tensor simulation. Medical Image Computing and Computer-Assisted Intervention - Miccai Pt 2 2006, 4191:718-725.

14. Fei BW, Duerk JL, Sodee DB, Wilson DL: Semiautomatic nonrigid registration for the prostate and pelvic MR volumes. Academic Radiology 2005, 12(7):815-824. 
15. Rueckert $D$, Hayes $C$, et al: Non-rigid registration of breast MR images using mutual information. Medical Image Computing and Computer-Assisted Intervention - Miccai 1998, 1496:1144-1152.

16. Rueckert $\mathrm{D}$, Sonoda $\mathrm{LI}$, et al: Nonrigid registration using free-form deformations: Application to breast MR images. IEEE Transactions on Medical Imaging 1999, 18(8):712-721.

17. Rohlfing $T$, Maurer $C R$, et al: Volume-preserving nonrigid registration of MR breast images using free-form deformation with an incompressibility constraint. IEEE Transactions on Medical Imaging 2003, 22(6):730-741.

18. Tanner C, Schnabel JA, et al: Quantitative evaluation of free-form deformation registration for dynamic contrastenhanced MR mammography. Medical Physics 2007, 34(4):1221-1233.

19. Froh MS, Barber DC, et al: Piecewise-quadrilateral registration by optical flow - Applications in contrast-enhanced MR imaging of the breast. Medical Image Computing and Computer-Assisted Intervention-Miccai Pt 2 2006, 4191:686-693.

20. Crum WR, Tanner $C$, et al: Anisotropic multi-scale fluid registration: evaluation in magnetic resonance breast imaging. Physics in Medicine and Biology 2005, 50(21):5153-5174.

21. Goshtasby A: Registration of Images with Geometric Distortions. IEEE Transactions on Geoscience and Remote Sensing 1988, 26(1):60-64.

22. Lapidus L, Pinder GF: Numerical Solution of Partial Differential Equations in Science and Engineering. WileyInterscience 1982.

doi:10.1186/1475-925X-9-8

Cite this article as: Ong et al: Non-rigid registration of breast surfaces using the laplace and diffusion equations. BioMedical Engineering OnLine 2010 9:8.

Submit your next manuscript to BioMed Central and take full advantage of:

- Convenient online submission

- Thorough peer review

- No space constraints or color figure charges

- Immediate publication on acceptance

- Inclusion in PubMed, CAS, Scopus and Google Scholar

- Research which is freely available for redistribution 